\title{
QUEEN'S
UNIVERSITY
BELFAST
}

\section{Second cancer in Philadelphia negative myeloproliferative neoplasms (MPN-K). A nested case-control study}

Barbui, T., Ghirardi, A., Masciulli, A., Carobbio, A., Palandri, F., Vianelli, N., De Stefano, V., Betti, S., Di Veroli, A., Iurlo, A., Cattaneo, D., Delaini, F., Bonifacio, M., Scaffidi, L., Patriarca, A., Rumi, E., Casetti, I. C., Stephenson, C., Guglielmelli, P., ... Finazzi, G. (2019). Second cancer in Philadelphia negative myeloproliferative neoplasms (MPN-K). A nested case-control study. Leukemia. https://doi.org/10.1038/s41375019-0487-8

Published in:

Leukemia

Document Version:

Peer reviewed version

Queen's University Belfast - Research Portal:

Link to publication record in Queen's University Belfast Research Portal

Publisher rights

(C) 2019 The Authors. This work is made available online in accordance with the publisher's policies. Please refer to any applicable terms of use of the publisher.

\section{General rights}

Copyright for the publications made accessible via the Queen's University Belfast Research Portal is retained by the author(s) and / or other copyright owners and it is a condition of accessing these publications that users recognise and abide by the legal requirements associated with these rights.

Take down policy

The Research Portal is Queen's institutional repository that provides access to Queen's research output. Every effort has been made to ensure that content in the Research Portal does not infringe any person's rights, or applicable UK laws. If you discover content in the Research Portal that you believe breaches copyright or violates any law, please contact openaccess@qub.ac.uk. 


\title{
Second cancer in Philadelphia negative Myeloproliferative neoplasms (MPN-K). A nested case-control study
}

\author{
Running title: Second cancer in MPN
}

Tiziano BARBUI ${ }^{1}$, Arianna GHIRARDI ${ }^{1}$, Arianna MASCIULLI ${ }^{1}$, Alessandra CAROBBIO $^{1}$, Francesca PALANDRI $^{2}$, Nicola VIANELLI ${ }^{2}$, Valerio DE STEFANO ${ }^{3}$, Silvia BETTI ${ }^{3}$, Ambra DI VEROLI ${ }^{4}$, Alessandra IURLO ${ }^{5}$, Daniele CATTANEO ${ }^{5}$, Federica DELAINI ${ }^{6}$, Massimiliano BONIFACIO ${ }^{7}$, Luigi SCAFFIDI ${ }^{7}$, Andrea PATRIARCA $^{8}$, Elisa RUMI ${ }^{9,10}$, llaria Carola CASETTI $^{9}$, Clemency STEPHENSON ${ }^{11}$, Paola GUGLIELMELLI $^{12}$, Elena Maria ELLI ${ }^{13}$, Miroslava PALOVA ${ }^{14}$, Laura BERTOLOTTII ${ }^{15}$, Daniel EREZ ${ }^{16}$, Montse GOMEZ ${ }^{17}$, Kai WILLE ${ }^{18}$, Manuel PEREZ-ENCINAS ${ }^{19}$, Francesca LUNGHI ${ }^{20}$, Anna ANGONA ${ }^{21}$, Maria Laura FOX ${ }^{22}$, Eloise BEGGIATO ${ }^{23}$, Giulia BENEVOLO ${ }^{24}$, Giuseppe CARLI ${ }^{25}$, Rossella CACCIOLA ${ }^{26}$, Mary Frances McMULLIN ${ }^{27}$, Alessia TIEGHI ${ }^{28}$, Valle RECASENS ${ }^{29}$, Monia MARCHETTI ${ }^{30}$, Martin GRIESSHAMMER $^{18}$, Alberto ALVAREZ-LARRAN ${ }^{31}$, Alessandro Maria VANNUCCHI ${ }^{12}$, Guido FINAZZI ${ }^{6}$

${ }^{1}$ FROM Research Foundation, Papa Giovanni XXIII Hospital, Bergamo, Italy

${ }^{2}$ Institute of Hematology "L. and A. Seràgnoli", S. Orsola-Malpighi Hospital, Bologna, Italy

${ }^{3}$ Institute of Hematology, Catholic University, Fondazione Policlinico Universitario A. Gemelli IRCCS, Roma, Italy

${ }^{4}$ Rete laziale MPN, Roma, Italy

${ }^{5}$ Hematology Division, Foundation IRCCS Ca' Granda Ospedale Maggiore Policlinico, and University of Milan, Milan, Italy

${ }^{6}$ Hematology Division, Papa Giovanni XXIII Hospital, Bergamo, Italy

${ }^{7}$ Department of Medicine, Section of Hematology, University of Verona, Italy

${ }^{8}$ Division of Hematology, Department of Translational Medicine, University of Eastern Piedmont, Novara, Italy

${ }^{9}$ Department of molecular Medicine, University of Pavia, Pavia, Italy

${ }^{10}$ Department of Hematology Oncology, Fondazione IRCCS Policlinico San Matteo Pavia, Italy

${ }^{11}$ Guy's and St Thomas' NHS Foundation Trust, London, UK

${ }^{12}$ CRIMM-Center of Research and Innovation of Myeloproliferative Neoplasms, Azienda

Ospedaliera Universitaria Careggi, Dept Experimental and Clinical medicine, and Denothe Center,

University of Florence, Italy

${ }^{13}$ Hematology Division, Ospedale San Gerardo, ASST Monza, Italy

${ }^{14}$ Department of Hemato-oncology, University Hospital Olomouc, Czech Republic

${ }^{15}$ S.C. Ematologia, Azienda Ospedaliera S. Croce e Carle, Cuneo, Italy

${ }^{16}$ Hematology Institute and Blood Bank, Meir Medical Center, Kfar Saba, and Sackler School of

Medicine Tel Aviv University, Israel

${ }^{17}$ Hematology Department, Hospital Clínico Universitario, Valencia, Spain

${ }^{18}$ University Clinic for Hematology and Oncology Minden, University of Bochum, Germany

${ }^{19}$ Hematology Department, Hospital Clínico Universitario de Santiago de Compostela, Spain 
${ }^{20}$ Hematology and Bone Marrow Transplantation Unit, IRCCS San Raffaele Scientific Institute, Milano, Italy

${ }^{21}$ Hematology Department, Hospital del Mar, Barcelona, Spain

${ }^{22}$ Hematology Department, Hospital Universitario Vall d'Hebron, Barcelona, Spain

${ }^{23}$ Unit of Hematology, Department of Oncology, University of Torino, Italy

${ }^{24}$ Hematology Division, Città della Salute e della Scienza Hospital, Torino, Italy

${ }^{25}$ Hematology Division, San Bortolo Hospital, Vicenza, Italy

${ }^{26}$ HAEMOSTASIS UNIT, Department of Clinical and Experimental Medicine, University of Catania, "Policlinico-Vittorio Emanuele" Hospital, Catania, Italy

${ }^{27}$ Queen's University, Belfast, UK

${ }^{28}$ Hematology Unit, Azienda Unità Sanitaria-IRCCS di Reggio Emilia, Italy

${ }^{29}$ Hematology Department, Hospital Universitario Miguel Servet, Zaragoza, Spain

${ }^{30}$ Oncology Unit, Cardinal Massaia Hospital, Asti, Italy

${ }^{31}$ Hematology Department, Hospital Clínic, Barcelona, Spain

\section{Competing Interests}

TB has been a speaker and consultant for Novartis and he has received research grant from AOP Orphan. VDS has received consulting and lecture fees from Amgen, Celgene, Novartis, and institutional research grants from Bayer and Novartis. MLF has been a member of advisory Board for Novartis and she has received travel grants from the company. MFM has been a speaker and consultant for Novartis. MM has received honoraria for advisory boards and lectures at sponsored meetings from Celgene, Amgen, Janssen, Gilead, Novartis. AMV has been a speaker for Novartis, Celgene and Shire and participated to advisory boards of Celgene, Incyte, Novartis.

The other authors declare no competing financial interests.

\section{Correspondence to:}

Tiziano Barbui, MD, Scientific Director, FROM Research Foundation, Papa Giovanni XXIII Hospital, Bergamo, Italy. Piazza OMS, 1 - 24127 Bergamo, Italy

Tel. +39(0)352675134 / +39(0)352674436; Fax: +39(0)352674926 
e-mail: tbarbui@fondazionefrom.it 


\begin{abstract}
We conducted a large international nested case-control study including 1,881 patients with Philadelphia-negative myeloproliferative neoplasms (MPN). Cases $(n=647)$ were patients with second cancer (SC: carcinoma, non-melanoma skin cancer, hematological second cancer and melanoma) and controls $(n=1,234)$ were patients without SC, matched with cases for sex, age at MPN diagnosis, date of MPN diagnosis and MPN disease duration.

The aim was to evaluate the risk of SC after exposure to cytoreductive drugs.

Patients exposed to hydroxyurea (HU) (median: 3 years) had a risk of SC similar to unexposed patients (OR=1.06, 95\% Cl 0.82-1.38). In contrast, in cancer-specific stratified multivariable analysis, HU had two-fold higher risk of non-melanoma (NM) skin cancer (OR=2.28, 95\% Cl 1.154.51). A significantly higher risk of NM-skin cancer was also documented for pipobroman $(\mathrm{OR}=3.74,95 \% \mathrm{Cl} 1.00$ - 14.01), ruxolitinib (OR=3.87, 95\% $\mathrm{Cl}$ 1.18-12.75) and for drug combination $(\mathrm{OR}=3.47,95 \% \mathrm{Cl}$ 1.55-7.75). These three drugs did not show excess risk of carcinoma and hematological second cancer compared with unexposed patients. Exposure to interferon, busulfan and anagrelide did not increase the risk.

In summary, while it is reassuring that no excess of carcinoma was documented, a careful dermatologic active surveillance before and during the course of treatments is recommended.
\end{abstract}




\section{Introduction}

Polycythemia vera (PV), essential thrombocythemia (ET) and myelofibrosis (MF) are myeloproliferative neoplasms (MPN) characterized by stem cell-derived clonal myeloproliferation with mutually exclusive driver $J A K 2, C A L R$, and MPL mutations and other sub-clonal somatic mutations.

Current management recommendations have brought benefits in decreasing the major complications of MPN diseases and have improved overall survival and quality of life [1]. However, the proportion of MPN patients with cardiovascular events, evolution to overt myelofibrosis and acute leukemia is still elevated and the mortality rate due to these causes is higher than in normal population $[2,3]$. While the risk of transforming to acute leukemia has been the subject of several studies [2] less is known about the risk of developing other malignancies such as solid tumors. In this regard, the available information derives from retrospective cohort analysis [4-10] and registry-based population studies $[11,12]$. These latter may have limitations as they do not have access to information on individual treatment, mutational status, and other important clinical features. The relationship between cytoreductive drugs and the development of these second malignancies has been poorly explored $[13,14]$. The only convincing demonstration of a carcinogenic potential of cytoreductive drugs has been provided for chlorambucil and radiophosphorus in a randomized clinical trial carried out in PV by the Polycythemia Vera Study Group (PVSG) [15], whereas the evidence that other cytoreductive agents including Hydroxyurea (HU), pipobroman, busulfan and JAK2 inhibitors, may affect the risk of second cancer (SC) in MPN remains uncertain.

This topic is complex also because MPNs themselves may have an intrinsic propensity to develop cancer $[12,16-18]$ making it difficult to distinguish the pathogenetic role of drug intervention from that of the MPN disease itself.

To tackle these relevant clinical questions, we designed a nested case-control study in the frame of European Leukemia Net (ELN). Cases were PV, ET and MF patients with SC, and controls were MPN patients who did not present or develop second malignancies during a comparable observation period. 
Our aim was twofold: (i) to describe the types and frequency of SC, including hematological second cancer (HSC) and (ii) to identify the risk-factors for SC, focusing on the role of cytoreductive agents. 


\section{Patients and Methods}

This multicenter international nested case-control study involved 30 international centers (18 from Italy, 11 from European centers and 1 from Israel) (ClinicalTrials.gov: NCT03745378).

For each center, patients with a diagnosis of Philadelphia-negative MPN according to PVSG, 2008 and 2016 WHO criteria (PV, ET, primary and secondary MF), performed from 2000 to 2016, were eligible for the study. Patients were excluded if any diagnosis of cancer occurred before the MPN diagnosis.

\section{Selection of cases and controls}

Cases were patients with SC diagnosed concurrently or subsequent to the diagnosis of MPN. Date of SC diagnosis was defined as index date. Controls were patients with a diagnosis of MPN without SC.

For each case with SC, up to 3 controls SC-free at the index date were matched by each center for sex, age at MPN diagnosis ( \pm 5 years), date of MPN diagnosis ( \pm 6 years) and MPN disease duration ( \pm 3 years). According to the nested case-control design, each set consisting of one case and their matched-controls had a similar observational period (from MPN diagnosis until the index date) and therefore the same chance to experience the outcome.

Cases without suitable controls were excluded from this analysis.

\section{Cytoreductive drugs exposure}

For each case and control, exposure to cytoreductive drugs was assessed during the whole period from MPN diagnosis and SC occurrence (for cases)/index date (for controls).

Exposure was defined as follows: (i) exposure to a single drug, irrespective of the line of treatment; (ii) exposure to a single drug in first-line monotherapy and (iii) exposure to more than one line of treatment with cytoreductive agents.

\section{Statistical analyses}

Descriptive statistics were used for summarizing the characteristics of cases and controls at MPN diagnosis and during follow-up. Categorical variables were presented as number and percentage; continuous variable as mean and standard deviation (SD) or median and interquartile range (IQR). 
Kruskal-Wallis test (for continuous variables) were used to test difference between SC cases with PV, ET and MF.

Chi-square test or Fisher's Exact test when appropriated (for categorical data) and t-test or MannWhitney $U$ test (for continuous variables) were used to test differences between cases and controls.

For each type of SC, cumulative incidence over time, from MPN diagnosis, was estimated by the Kaplan-Meier method and were compared in PV, ET and MF using the log-rank test.

Conditional logistic regression models were fitted to estimate the Odds Ratio (OR) of SC (overall and stratified by the type of tumor), as well as the corresponding 95\% Confidence Interval (Cl), associated with cytoreductive exposure according to definition (i)-(iii). Adjustments were made for the characteristics of patients at MPN diagnosis such as age, type of MPN, duration of MPN disease, genetics data, aspirin use and the occurrence of major thrombosis and monoclonal gammopathy of uncertain significance (MGUS) during follow-up.

A sensitivity analysis was performed to evaluate the robustness of our findings by excluding SC cases (and their corresponding matched controls) without follow-up, as they were diagnosed concurrently with the MPN diagnosis.

For all hypotheses tested, two-tailed p-values less than 0.05 were considered to be significant.

Analyses were performed using STATA software, release 13 (StataCorp LP, College Station TX, USA).

\section{Results}

\section{A. Cases}

Cases with SC, stratified by type of MPN, are described in Table 1.

Median time to SC occurrence from MPN diagnosis was 4.5 years and the most frequent category (65.8\%) was represented by carcinoma, especially of the prostate, breast, colon-rectum, and lung. A trend for a higher frequency of carcinoma in patients with ET and PV than MF was observed $(p=0.079)$. The percentage of cases with NM-skin cancers, HSC and melanoma did not differ in the three MPNs. We underscore that a higher proportion of patients with MF presented with 
carcinoma closer to diagnosis ( $p=0.062$ ); moreover, in $25.8 \%$ of patients, HSC were synchronous with MPN diagnosis with a prevalence of indolent non-Hodgkin lymphoma and CLL (10 out of 16 patients). On the contrary, the synchronous occurrence was seen in only $3-4 \%$ of cases with melanoma and carcinoma and in none of patients with skin cancer. Of note, the appearance of SC in MF was persistently earlier than in PV and ET as shown in Figure 1 which illustrates the cumulative incidence over time of the single tumor category.

\section{B. Cases and matched-controls}

Cases with SC were comparable to controls for demographics, type of MPN, and exposure to potential baseline clinical and laboratory confounders such as driver and non-driver mutations, abnormal karyotype and prior cardiovascular risk factors (Table 2). Of note, the proportion of patients developing major arterial thrombosis and monoclonal gammopathy of uncertain significance (MGUS) was significantly more elevated in cases than in controls ( $p=0.015$ and 0.005 , respectively). No significant association was found for driver and non-driver mutations and SC.

In detail, major thrombosis after MPN diagnosis and before SC occurrence was significantly more frequent in patients with carcinoma $(p=0.034)$ while the occurrence of MGUS preceding cancer was higher among cases with HSC $(p=0.000)$, in particular in patients with multiple myeloma (33.3\%) (Table S1). Interestingly, while in PV there was a trend for a higher frequency of melanoma $(p=0.077)$ and carcinoma $(p=0.065)$, the proportion of MF was significantly higher among cases with NM-skin cancers than controls $(p=0.040)$.

\section{Drug exposure}

About $21.3 \%$ of cases with SC and $25.3 \%$ controls remained chemotherapy-free during the followup period (Table 3 and Table S2).

Hydroxyurea The percentage of cases treated with $\mathrm{HU}$ during the period from diagnosis to SC occurrence, regardless of the lines of therapy, was similar to that of controls $(p=0.793)$ as well as when the drug was used alone in monotherapy $(p=0.310)$. These findings were confirmed in a multivariable analysis showing that patients treated with $\mathrm{HU}$, regardless of the line of treatment or 
in first-line monotherapy, had a similar SC risk than patients who were not treated with this drug (OR=1.06, 95\% $\mathrm{Cl} 0.82-1.38$ and $\mathrm{OR}=1.14,95 \% \mathrm{Cl} 0.84-1.54)$ (Figure 2).

However, in cancer-specific stratified multivariable analysis, HU irrespective of the line of treatment, showed a two-fold higher risk of NM-skin cancer (OR=2.28, 95\% Cl 1.15-4.51) (Figure S1).

Moreover, a statistically significant protection of HU alone in first-line monotherapy on risk of HSC was found (Figure S1), even though in a sensitivity analysis (see below) this finding was not confirmed.

Pipobroman The proportion of SC cases treated with pipobroman, irrespective of the line of treatment, was significantly higher than controls $(p=0.018)$ while a trend was seen for the exposure in first-line monotherapy ( $p=0.061$ ) (Table 3). Notably, in multivariable analysis, the overall exposure to pipobroman, was significantly associated with the risk of SC $(\mathrm{OR}=2.10,95 \% \mathrm{Cl}$ 1.09-4.06) (Figure 2), especially with NM-skin cancer (OR=3.74, 95\% Cl 1.00 - 14.01) (Figure S1).

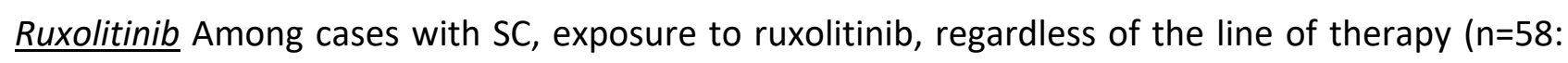
$n=35 M F, n=6 E T, n=17 P V)$ and alone in first-line monotherapy ( $n=17: n=16 M F$ and $n=1 E T)$, was significantly higher than controls ( $p=0.048$ and $p=0.033$, respectively). In the multivariable analysis, the association of ruxolitinib with SC was confirmed, particularly when patients were treated as first-line monotherapy for $M F(O R=4.00,95 \% \mathrm{Cl}$ 1.23-13.06) while a trend towards a higher risk of SC was observed when the exposure to this drug was considered irrespective of the line of treatment $(\mathrm{OR}=1.68,95 \% \mathrm{Cl}$ 0.92-3.09) (Figure 2). Of note, the higher risk of SC in patients exposed to ruxolitinib was limited to NM-skin cancers in which the risk was almost 4-fold higher than in non-exposed (OR=3.87, 95\% Cl 1.18-12.75) (Figure S1).

Drug combination Exposure to multiple cytoreductive drugs in combination or sequentially was more frequent in cases than in controls $(p=0.038)$ (Table 3). However, this univariate result was not confirmed in multivariable analysis (Figure 2 and S1) that showed that the exposure to multiple drugs did not increase the risk of overall SC but only the risk of NM-skin cancer (OR=3.47, 95\% Cl 1.55-7.75).

IFN, busulfan or anagrelide The proportion of patients treated with these drugs did not differ in comparison with controls (Table 3 ). No association with the risk of overall SC was observed in multivariable model (Figure 2). However, as reported in the recent guidelines, caution should be employed in prescribing busulfan that is indicated at low intermittent dose in elderly [1]. 


\section{Sensitivity analysis}

To evaluate the robustness of our findings we excluded from the analysis the 35 cases with SC (and their matched controls, $n=68$ ) without follow-up as they were diagnosed concurrently with MPN diagnosis.

We confirmed that the drugs increasing the risk of SC were pipobroman $(\mathrm{OR}=2.10,95 \% \mathrm{Cl}$ 1.094.06), when considered irrespective of the line of treatment, ruxolitinib (OR=5.24, 95\% $\mathrm{Cl} 1.47$ 18.71) in first-line monotherapy and the exposure to multiple cytoreductive agents in different lines of treatment $(\mathrm{OR}=1.46,95 \% \mathrm{Cl} 1.02-2.09)$.

In the stratified analysis according to the type of SC, the lack of association between any cytoreductive drug and carcinoma was confirmed.

In contrast, the significant protection of HU on HSC found in the primary multivariable analysis was not confirmed when the 16 cases with concurrent hematological cancer (and their 31 matched controls) were excluded.

\section{Discussion}

The first aim of this paper was to describe second cancers (SC) observed in clinical practice of contemporaneous patients with MPN. Time of SC emergence from diagnosis was shorter in MF as compared to ET and PV and this pattern was maintained during the follow-up from diagnosis to the development of SC. Of note, in a quarter of cases, diagnosis of MPN was synchronous with hematological cancer revealing an intrinsic predisposition even in untreated MPN patients [16-18]. Therefore, although the association of MPN and SC in the same patient is a relatively uncommon event, a careful surveillance of possible co-existence of hematological malignancy in MF from the beginning of diagnosis may be indicated. A number of evidences indicate that inflammation and deranged innate immunity intervene in the pathobiology of MF at the same extent as in solid cancers [18]. Although this remains entirely speculative at present time, yet several lines of evidences indicate that, among MPN, cells from MF patients are those characterized by more overtly deranged reactive oxygen species levels and abnormal DNA repair properties [18]. In addition, the germline telomerase reverse transcriptase (TERT) rs2736100_C variant found in MPN, was identified as a susceptibility factor for a variety of solid tumors [19] and recently it has been hypothesized that co-occurrence of MPN and cancer might be attributed to a synergism between cytoreductive therapy and TERT polymorphism [20]. 
Cases and controls were comparable for baseline clinical and laboratory characteristics. Interestingly, the proportion of patients developing major arterial thrombosis and of those presenting with MGUS during the observation from diagnosis was significantly greater in cases than controls. This latter finding is in line with recent data reporting that the presence of MGUS in PV and ET leads to poor prognosis due to second malignancy including non-hematological solid tumors [21]. Regarding the role of thrombosis, the association of cancer with venous thromboembolism is well known [22,23]. Conversely, the association between arterial thromboembolism and occult cancer is less certain. Few studies have systematically analyzed the association between cancer and preceding arterial thromboembolic events [24]. A very recent study evaluated the risk of arterial thromboembolism before cancer diagnosis in a series of 374,331 patients with age higher than 67 years. In these non MPN cases, the risk of arterial thromboembolic events was increased up to $69 \%$ in the year before cancer diagnosis [25]. A possible biological reason for the link between arterial thrombosis and SC may be related to an underlying common pathogenetic mechanism. It has been proposed that an aberrant inflammatory response consistently found in MPN may precede both thrombosis and second cancer [18].

The second aim of our study was to investigate the pathogenetic role of cytoreductive drugs used to control the chronic phase of PV, ET, and MF. The exposure was evaluated for each of the single drugs regardless of the line of treatment, in only the first line and in association with more cytoreductive drugs.

$\mathrm{HU}$ was the agent most frequently prescribed and the percentage of exposed cases was not different from that of controls, considering overall SC groups. However, when the model was fitted in the single SC categories, HU was significantly associated with the appearance of nonmelanoma skin cancer confirming previous uncontrolled observations $[26,27]$ and registry data [12]. It is reassuring that, our findings did not confirm a previous report [28] that HU increases the risk of more serious cancers such as carcinoma. Similar results were obtained in a propensity score analysis of a large number of PV patients enrolled in the European Collaborative Aspirin Study (ECLAP) in which HU exposure did not enhance the carcinoma risk compared with phlebotomy alone, although the median observational period was only 2.7 years [7]. 
As $\mathrm{HU}$ continues to have a prominent role in the treatment of PV, ET and MF, clinicians must be aware that the exposure to this drug in our study was limited to a median period of 3 years (range $1.2-6.1$ ) and, with this exposure duration, $\mathrm{HU}$ did not increased the risk of melanoma, carcinoma and HSC. Moreover, we underscore that previous reports indicate that exposure to HU at any cumulative dose level did not increase the risk of AML/MDS transformation neither in a crude analysis nor after adjustments for other treatments [29]. However, before reaching a firm conclusion in this regard, a longer follow-up is warranted.

Patients treated with pipobroman developed SC in a greater percentage than controls and, in multivariable analysis, the potential of this drug to enhance the risk was statistically significant when administered irrespective of the line of treatment (OR=2.10 (95\% Cl\% 1.09-4.06). This and other findings reporting a leukemogenic action of this drug [30] reinforce the recommendation that it should no longer be used in the treatment of MPN.

The effect of ruxolitinib on the occurrence of SC was evaluated in 58 patients, 27 cases (4.2\%) and 31 controls $(2.5 \%)(p=0.048)$, regardless of the line of treatment and in 17 patients treated with the drug alone in first-line monotherapy, 10 cases $(1.5 \%)$ and 7 controls $(0.6 \%) \quad(p=0.033)$. ruxolitinib exposure was higher in cases with SC than in controls and the increased risk when the drug was used in first-line monotherapy, was confirmed in multivariable analysis (OR 4.00, $95 \% \mathrm{Cl}$ 1.23-13.06) after adjustment for age, duration of the MPN disease, type of MPN, JAK2 mutation, appearance of a thrombotic event and MGUS during-follow-up. This was also shown when the multivariable analysis was performed in patients exposed to ruxolitinib independently of the line of treatment although only a trend to enhanced risk was documented (OR=1.68, $95 \% \mathrm{Cl} 0.92$ 3.09). Types of SC associated with ruxolitinib were exclusively non-melanoma skin cancer suggesting that this JAK1.2 inhibitor may act as immunosuppressive agent. Of note, no excess of cases with HSC including aggressive NHL in comparison with controls was documented in our series. However, this latter finding in a limited number of patients, cannot refute the recently reported results of an increased risk of NHL [31].

The hypothesis that ruxolitinib exposure may be associated with increased risk of developing cancer must be taken with great caution given the limited number of patients, but represents a signal to suggest appropriate strict pharmacovigilance programs. For the time being, the awareness that these malignancies may occur in patients receiving ruxolitinib even in in the absence of previous exposure to other cytoreductive drugs, should not influence the 
recommendations on its use, also because these second cancers are non-aggressive and treatable. Clearly, a careful clinical and laboratory surveillance is indicated.

The limitations of our study are intrinsic to the retrospective design and to possible residual confounders, which might have influenced the occurrence of outcomes. Moreover, a bias related to the limited follow-up and, consequently, to the limited exposure time to drugs as well as a selection bias of cases and controls cannot be excluded.

However, we highlight that this nested case-control study performed in rare diseases such as MPN, represents the most efficient way, among retrospective studies, to identify the association between exposure to drugs and outcomes especially when they occur after long observation time. Moreover, another strength of this survey is the large number of patients recruited and followedup in a qualified network of 30 European hematological centers.

In summary, we did not show evidence that $\mathrm{HU}$, administered as single drug, contributes to the occurrence of melanoma, carcinoma and HSC, while we confirm that this agent, may increase the risk of non-melanoma skin cancers. Similarly, MF patients exposed to ruxolitinib alone in first-line monotherapy or in association with other cytoreductive agents may be prone to a higher probability of non-melanoma skin cancer. Accordingly, for the time being, we recommend active dermatological surveillance before and during these therapies.

These findings urgently needs to be confirmed in appropriate studies involving larger series of patients with an adequate follow-up.

\section{Acknowledgments}

This study was supported by the FROM Research Foundation, Papa Giovanni XXIII Hospital, Bergamo, Italy. The study was also supported by Associazione Italiana per la Ricerca sul Cancro, grant 5perMille, progetto MYNERVA, to PG and AMV.

\section{Competing Interests}

TB has been a speaker and consultant for Novartis and he has received research grant from AOP Orphan. VDS has received consulting and lecture fees from Amgen, Celgene, Novartis, and institutional research grants from Bayer and Novartis. MLF has been a member of advisory Board for Novartis and she has received travel grants from the company. MFM has been a speaker and 
consultant for Novartis. MM has received honoraria for advisory boards and lectures at sponsored meetings from Celgene, Amgen, Janssen, Gilead, Novartis. AMV has been a speaker for Novartis, Celgene and Shire and participated to advisory boards of Celgene, Incyte, Novartis.

The other authors declare no competing financial interests.

\section{Authorship}

TB designed the study, collected the data, interpreted the results and wrote the paper. AG collected the data, performed the statistical analysis and wrote the paper. AMV, VDS, GF and MM interpreted the results and wrote the paper. The final version of the manuscript was approved by all the other authors. 


\section{References}

1. Barbui T, Tefferi A, Vannucchi AM, Passamonti F, Silver RT, Hoffman R, et al. Philadelphia chromosome-negative classical myeloproliferative neoplasms: revised management recommendations from European LeukemiaNet. Leukemia 2018; 32: 1057-1069.

2. Tefferi A, Pardanani A. Myeloproliferative Neoplasms: A Contemporary Review. JAMA Oncol 2015; 1: 97-105.

3. Barbui T, Carobbio A, Rumi E, Finazzi G, Gisslinger H, Rodeghiero F, et al. In contemporary patients with polycythemia vera, rates of thrombosis and risk factors delineate a new clinical epidemiology. Blood 2014; 124: 3021-3023.

4. Chattopadhyay S, Zheng G, Sud A, Yu H, Sundquist K, Sundquist J, et al. Risk of second primary cancer following myeloid neoplasia and risk of myeloid neoplasia as second primary cancer: a nationwide, observational follow up study in Sweden. Lancet Haematol 2018; 5: e368-e377.

5. Vannucchi MA, Masala G, Antonioli E, Susini MC, Guglielmelli P, Pieri L, et al. Increased risk of lymphoid neoplasms in patients with Philadelphia chromosome-negative myeloproliferative neoplasms. Cancer epidemiol biomarkers prev 2009; 18: 2068-2073.

6. Marchetti M, Carobbio A, Capitoni E, Barbui T. Lymphoproliferative disorders in patients with chronic myeloproliferative neoplasms: A systematic review. Am J Hematol 2018; 93: 698-703.

7. Ghirardi A, Carobbio A, Masciulli A, Barbui T. Incidence of solid tumors in polycythemia vera treated with phlebotomy with or without hydroxyurea: ECLAP follow-up data. Blood Cancer J 2018; 8: 5.

8. Rumi E, Passamonti F, Elena C, Pietra D, Arcaini L, Astori C, et al. Increased risk of lymphoid neoplasm in patients with myeloproliferative neoplasm: a study of 1,915 patients. Haematologica 2011; 96: 454-458.

9. Kissova J, Ovesna P, Penka M, Bulikova A, Kiss I. Second malignancies in philadelphianegative myeloproliferative neoplasms-single-center experience. Anticancer Res 2014; 34: 2489-2496.

10. Birgegård G, Folkvaljon F, Garmo H, Holmberg L, Besses C, Griesshammer M, et al. Leukemic transformation and second cancers in 3649 patients with high-risk essential thrombocythemia in the EXELS study. Leuk Res 2018; 74: 105-109.

11. Frederiksen H, Farkas DK, Christiansen CF, Hasselbalch HC, Sorensen HT. Chronic myeloproliferative neoplasms and subsequent cancer risk: a Danish population-based cohort study. Blood 2011; 118: 6515-6520.

12. Landtblom AR, Bower H, Andersson TM, Dickman PW, Samuelsson J, Björkholm M, et al. Second malignancies in patients with myeloproliferative neoplasms: a population-based cohort study of 9379 patients. Leukemia 2018; 32: 2203-2210. 
13. Masarova L, Cherry M, Newberry KJ, Estrov Z, Cortes JE, Kantarjian HM, et al. Secondary solid tumors and lymphoma in patients with essential thrombocythemia and polycythemia vera single center experience. Leuk Lymphoma 2016; 57: 237-239.

14. Marcheselli L, Polliack A, Tadmor T. Impact of therapy on the development of second malignancies in essential thrombocythemia and polycythemia vera: Are we comfortable about this? Leuk Lymphoma 2016; 57: 6-7.

15. Berk PD, Goldberg JD, Donovan PB, Fruchtman SM, Berlin NI, Wasserman LR. Therapeutic recommendations in polycythemia vera based on Polycythemia Vera Study Group protocols. Semin Hematol 1986; 23: 132-143.

16. Pettersson $\mathrm{H}$, Knutsen $\mathrm{H}$, Holmberg $\mathrm{E}$, Andréasson $\mathrm{B}$. Increased incidence of another cancer in myeloproliferative neoplasms patients at the time of diagnosis. Eur J Haematol 2015; 94: 152-156.

17. Gaidano G, Guerrasio A, Serra A, Rege-Cambrin G, Saglio G. Molecular mechanisms of tumor progression in chronic myeloproliferative disorders. Leukemia 1994; 8(Suppl 1): S27-S29.

18. Hasselbalch HC. Chronic inflammation as a promotor of mutagenesis in essential thrombocythemia, polycythemia vera and myelofibrosis. A human inflammation model for cancer development? Leuk Res 2013; 37: 214-220.

19. Jäger R, Harutyunyan AS, Rumi E, Pietra D, Berg T, et al. Common germline variation at the TERT locus contributes to familial clustering of myeloproliferative neoplasms. Am J Hematol 2014; 89: 1107-1110.

20. Krahling T, Balassa K, Kiss KP, Bors A, Batai A, Halm G, et al. Co-occurrence of Myeloproliferative Neoplasms and Solid Tumors Is Attributed to a Synergism Between Cytoreductive Therapy and the Common TERT Polymorphism rs2736100. Cancer Epidemiol Biomarkers Prev 2016; 25: 98-104.

21. Le Clech L, Sakka M, Meskar A, Kerspern H, Eveillard JR, Berthou C, et al. The presence of monoclonal gammopathy in $\mathrm{Ph}$-negative myeloproliferative neoplasms is associated with a detrimental effect on outcomes. Leuk Lymphoma 2017; 58: 2582-2587.

22. White RH, Chew HK, Zhou H, Parikh-Patel A, Harris D, Harvey D, et al. Incidence of venous thromboembolism in the year before the diagnosis of cancer in 528,693 adults. Arch Intern Med 2005; 165: 1782-1787.

23. Streiff MB. Thrombosis in the setting of cancer. Hematology Am Soc Hematol Educ Program 2016; 2016: 196-205.

24. De Stefano V. Arterial thrombosis and cancer: the neglected side of the coin of Trousseau syndrome. Haematologica 2018; 103: 1419-1421.

25. Navi BB, Reiner AS, Kamel H, ladecola C, Okin PM, Tagawa ST, et al. Arterial thromboembolic events preceding the diagnosis of cancer in older persons. Blood 2019; 133: 781-789.

26. Sanchez-Palacios C, Guitart J. Hydroxyurea associated squamous dysplasia. J Am Acad Dermatol 2004; 51: 293-300. 
27. Antonioli E, Guglielmelli P, Pieri L, Finazzi M, Rumi E, Martinelli V, et al. Hydroxyurea-related toxicity in 3,411 patients with Ph'-negative MPN. Am J Hematol 2012; 87: 552-554.

28. Najean $\mathrm{Y}$, Rain JD. Treatment of polycythemia vera: use of 32P alone or in combination with maintenance therapy using hydroxyurea in 461 patients greater than 65 years of age. The French Polycythemia Study Group. Blood 1997; 89: 2319-2327.

29. Björkholm M, Derolf AR, Hultcrantz M, Kristinsson SY, Ekstrand C, Goldin LR, et al. Treatment-related risk factors for transformation to acute myeloid leukemia and myelodysplastic syndromes in myeloproliferative neoplasms. J Clin Oncol 2011;29:24102415.

30. Kiladjian JJ, Chevret S, Dosquet C, Chomienne C, Rain JD. Treatment of polycythemia vera with hydroxyurea and pipobroman: final results of a randomized trial initiated in 1980 . J Clin Oncol 2011; 29: 3907-3913.

31. Porpaczy E, Tripolt S, Hoelbl-Kovacic A, Gisslinger B, Bago-Horvath Z, Casanova-Hevia E, et al. Aggressive B-cell lymphomas in patients with myelofibrosis receiving JAK1/2 inhibitor therapy. Blood 2018; 132: 694-706. 


\section{FIGURE LENGENDS}

\section{Figure 1. Time to different second cancer (SC) in each MPN disease (only for case patients)}

NM-skin cancer: Non-melanoma skin cancer; HSC: Hematological Second Cancer.

Figure 2. Effect of cytoreductive drugs (according to (i) exposure to a single drug, irrespective of the line of treatment, (ii) exposure to a single drug as first-line monotherapy and (iii) exposure to more than one line of treatment) on cancer risk estimated by a multivariable conditional logistic regression model adjusted for potential confounders

OR: Odds Ratio; Cl: Confidence Interval

(i) Single drug exposure during follow-up (at least one administration regardless of the line of treatment): patients could be exposed to more than one cytoreductive drug during follow-up; (iii) Single drug exposure during follow-up as a first-line monotherapy and (iii) exposure to multiple treatment lines with cytoreductive drugs.

*Significant association with second cancer risk ( $p<0.05)$; The model was adjusted for age at MPN diagnosis, type of MPN diagnosis (ET/PV/MF), JAK2 status, duration of MPN disease, occurrence thrombosis and MGUS during follow-up and aspirin use. 


\section{TABLES}

\section{Table 1. Type and staging of second cancer (SC) included in the study}

NM-skin cancer: Non-melanoma skin cancer; HSC: Hematological Second Cancer; IQR: Interquartile Range ${ }^{*} K r u s k a l-W a l l i s ~ t e s t$ for continuous variables, chi-square test or Fisher's exact test (when appropriate) for categorical data.

Table 2. Characteristics of 647 cases of second cancer (SC) and 1,234 controls at MPN diagnosis and major events during follow-up (before SC/index date)

IQR: Interquartile Range: mv: matching variable

${ }^{*} T$-test for continuous variables, chi-square test for categorical data; ${ }^{* *}$ Tested in 142 patients.

Table 3. Treatment of patients after MPN diagnosis and before second cancer (SC)/index date

${ }^{*}$ Chi-square test or Fisher's exact test (when appropriate) for categorical data

${ }^{* *}$ (i) Single drug exposure during follow-up (at least one administration regardless of the line of treatment): patients could be exposed to more than one cytoreductive drug during follow-up; (iii) Single drug exposure during follow-up as a first-line monotherapy and (iii) exposure to multiple treatment lines with cytoreductive drugs. 